\title{
ZigBee-Based Wireless Sensor Network for Temperature Monitoring
}

Dr. Anas Ali Hussien, Ali Hameed Tawfeeq

Lecturer, Department of Computer Engineering, Alnahrain University, Baghdad, Iraq anasali78@yahoo.com

MSc Research Student, Department of Computer Engineering, Alnahrain University, Baghdad, Iraq

\section{ABSTRACT}

ali.ziara@gmail.com

The proposed system in this paper describes the design and implementation of a Wireless Sensor Network (WSN) based on ZigBee technology to monitor the history and current temperature information of remote locations. The system is an electronic embedded system that contains: Base Station with coordinator ZigBee, End nodes with router ZigBee, and host computer as a data base (DB) server. The base station is responsible for data collecting, a display for end node sensor readings, and TCP/IP communication with the host computer. End nodes are directly connected with sensors and transmitting the readings of these sensors to the base station. The host computer is responsible for building a data base from end nodes sensor readings. The Microcontroller Units (MCUs) from Peripheral Interface Controller (PIC) family have been used and the firmware has been developed using $C$ language with MikroC Pro which is a full-featured ANSI C Compiler for Microchip PIC MCU as a development environment.

\section{Indexing terms/Keywords}

Wireless Sensor Network, IEEE 802.15.4 ZigBee, PIC Microcontrollers, Temperature Monitoring

\section{SUBJECT CLASSIFICATION}

Ad Hoc Wireless Sensor Network

\section{Council for Innovative Research}

Peer Review Research Publishing System

Journal: INTERNATIONAL JOURNAL OF COMPUTERS \& TECHNOLOGY

Vol 10, No 10 


\section{INTRODUCTION}

Nowadays, there are many challenges in remote locations environment monitoring. In the same time there are many technologies that can be used to resolve these challenges. Wireless Sensor Networks (WSN) is the technology that could provide ubiquitous computing [1]. Rapid advances in the areas of sensor design, information technologies, and wireless networks have paved the way for the proliferation of wireless sensor networks[2]. A sensor network is an infrastructure comprised of sensing (measuring), computing, and communication elements that gives an administrator the ability to instrument, observe, and react to events and phenomena in a specified environment[3].

ZigBee specification defines ZigBee's smart, cost-effective and energy-efficient mesh network. ZigBee technology is a low data rate, low power consumption, low cost; wireless networking protocol targeted towards automation and remote control applications. Table 1 shows WIFI, Bluetooth, and ZigBee main features comparison.

Table 1: WIFI, Bluetooth, and ZigBee main features comparison

\begin{tabular}{|c|c|c|c|}
\hline Feature & IEEE 802.11b & Bluetooth & ZigBee \\
\hline Power & Hours & Days & Simple \\
\hline Complexity & Very Complex & Complex & 64000 \\
\hline Nodes/Master & 32 & 7 & $30 \mathrm{~ms}-1 \mathrm{~s}$ \\
\hline Latency & $3 \mathrm{~s}$ & $10 \mathrm{~s}$ & $70 \mathrm{~m}-300 \mathrm{~m}$ \\
\hline Range & $100 \mathrm{~m}$ & No & Yes \\
\hline Extendibility & Rooming possible & $1 \mathrm{Mbps}$ & $250 \mathrm{Kbps}$ \\
\hline Data Rate & 11Mbps & $64 \mathrm{bit}, 128 \mathrm{bit}$ & $128 \mathrm{bit}$ AES \\
\hline Security & CCMP/TKIP 128bit/64bit & & \\
\hline
\end{tabular}

The IEEE 802.15.4 standard operates in the $868 \mathrm{MHz}, 915 \mathrm{MHz}$, and $2.45 \mathrm{GHz}$ frequency bands. The data rates supported by this standard are 20, 40, and $250 \mathrm{kbps}$ [4]. The air interface is Direct Sequence Spread Spectrum (DSSS) using Binary Phase Shift Keying (BPSK) for $868 \mathrm{MHz}$ and $915 \mathrm{MHz}$ and Offset Quadrature Phase-Shift Keying (O-QPSK) for $2.4 \mathrm{GHz}$. With the emergence of the ZigBee/IEEE 802.15.4 standard, systems are expected to transition to standardsbased approaches, allowing sensors to transfer information in a standardized manner[3]. ZigBee devices are the combination of application (such as light sensor, lighting control etc), ZigBee logical (coordinator, router, end device), and ZigBee physical device types (Full Function Device and Reduced Function Device)[4] (see figure 1-a). There are three network topologies considered in IEEE 802.15.4: star, tree network, and mesh, (see figure 1-b).

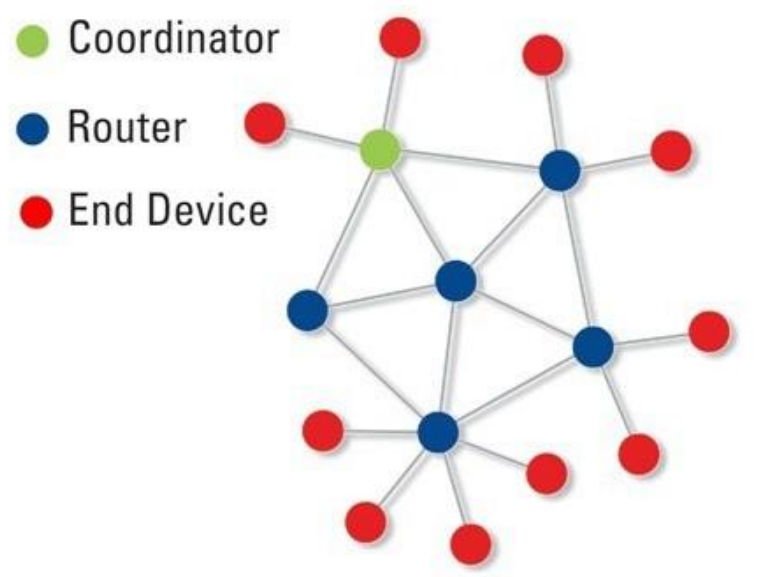

(a)

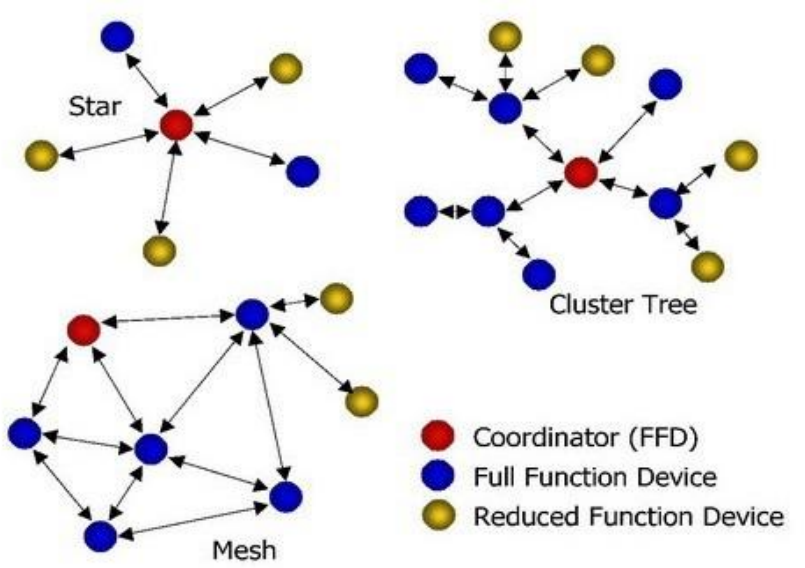

(b)

Fig 1: (a) ZigBee network devices (b) ZigBee network topologies

IEEE 802.15 .4 supports both short (16-bit) and extended (64-bit) addressing. An extended address (also called EUI-64) is assigned to every RF module that complies with the 802.15.4 specification. When a device associates with a Wireless Personal Area Network (WPAN) it can receive a 16 bit address from its parent node that is unique in that network. Every ZigBee network has a 16-bit PAN ID which identifies the network. By the use of the PAN IDs more than one network can 
exist on the same channel. The PAN ID is sent together with all packets. This means that devices can discard packets which are meant for another network[5].

Figure 2 shows IEEE 802.15.4 protocol architecture which focuses on the specification of the lower two layers of the protocol (physical and data link layer). On the other hand, ZigBee Alliance aims to provide the upper layers of the protocol stack (from network to the application layer) for interoperable data networking, security services and a range of wireless home and building control solutions, provide interoperability compliance testing, marketing of the standard, advanced engineering for the evolution of the standard. 802.15.4 itself defines the physical and MAC layers, whereas ZigBee defines the network and application layers[6].

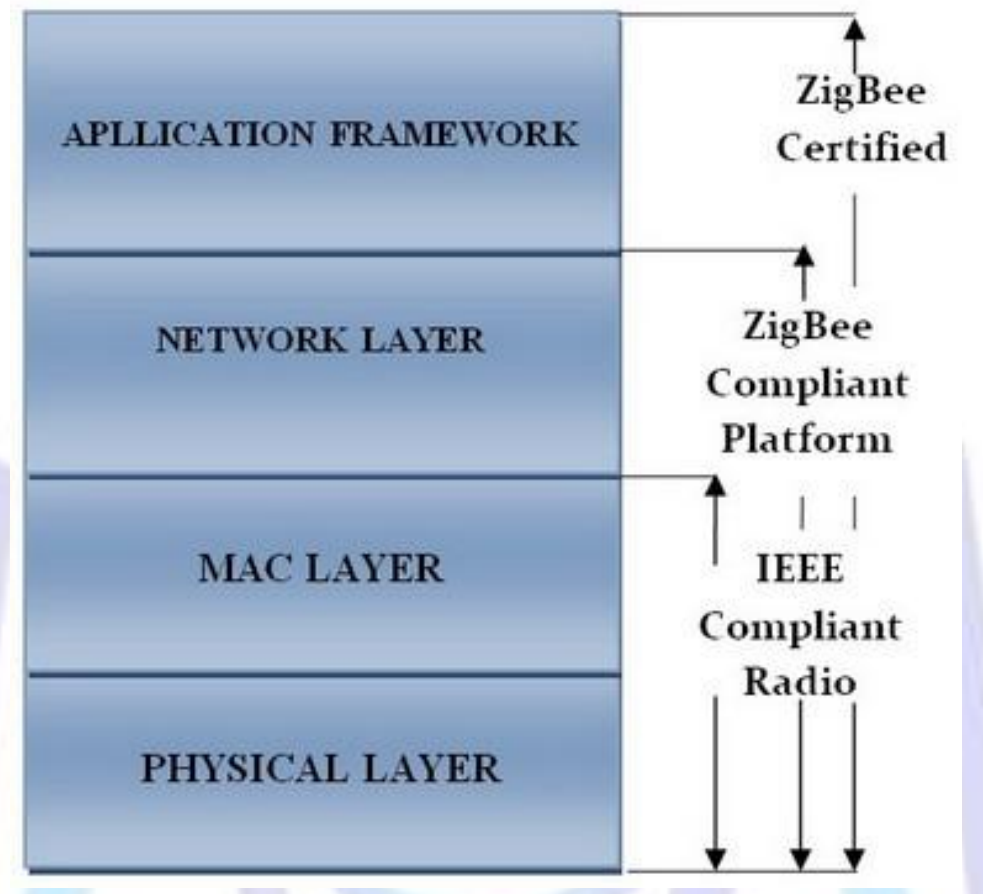

Fig 2: ZigBee protocol architecture

\section{SYSTEM HARDWARE PLATFORM}

A block diagram for the proposed system is shown in figure 3. Each of End Nodes communicates with the Base Station which will display the sensor information received from end nodes on LCD and send this information to the server to be saved into an SQL database as history information. An evaluating hardware has been developed with the intention to build our proposed system which included two main devices: Base Station and End node.

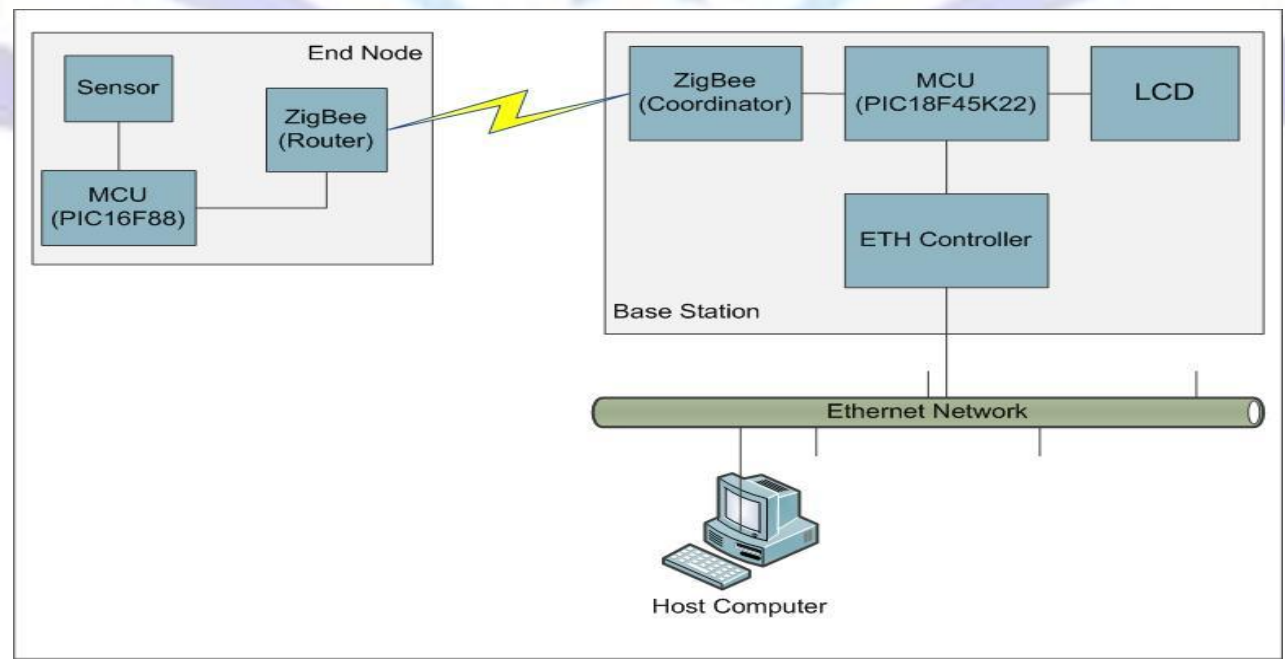

Fig 3: Proposed ZigBee System 


\section{Base Station Hardware Platform}

\section{Microcontroller}

A high performance microcontroller PIC18F45K22 as shown in figure (4-a) has been chosen to be the core and main processor for our proposed base station. PIC18F45K22 microcontroller from Microchip has many device specifications that served our proposed system [6]. The main features that concern the system are the Flash Memory size, RAM size, CPU speed, and Digital communication peripherals as the system has used UART interface with ZigBee module and SPI interface with Ethernet controller module plus Ethernet protocol stack has been loaded on the MCU.

\section{ZigBee Chip}

The ZigBee Chip CC2530 from Texas Instrument [7] and integrated into DRF1605 board as shown in figure (4-b) [8] has been chosen to provide the ZigBee protocol communication between the Base Station and End Nodes. DRF1605 ZigBee module provide serial UART interface for direct send/receive data communication with microcontrollers. This module to be used in the Base Station, it has been configured as a Coordinator with a specific PAN ID using the software application provided with the module.

\section{LCD Module}

A 2x16 LCD (1602A) shown in figure (4-c) with 4-bit interface mode [9] has been chosen to display the sensor readings received from end nodes.

\section{Ethernet Controller}

The Ethernet connection with the data base server has been done using an ETH Click module from MikroElekronik. This module features ENC28J60, a 28-pin 10Base-T standalone Ethernet controller, 8 Kbytes of Buffer RAM and SPI serial interface [10]. See Figure (4-d).

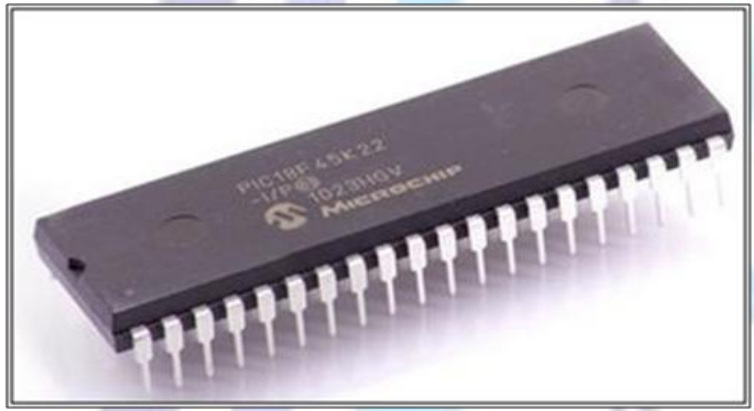

(a) PIC18F45K22 Microcontroller

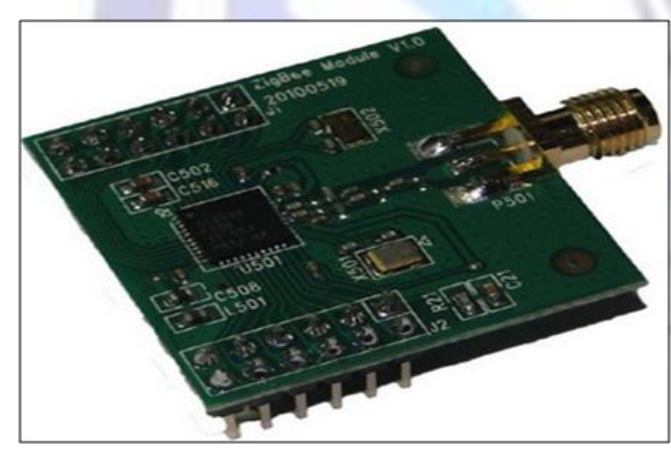

(b) DRF1605 ZigBee Module



(b) $2 \times 16$ LCD

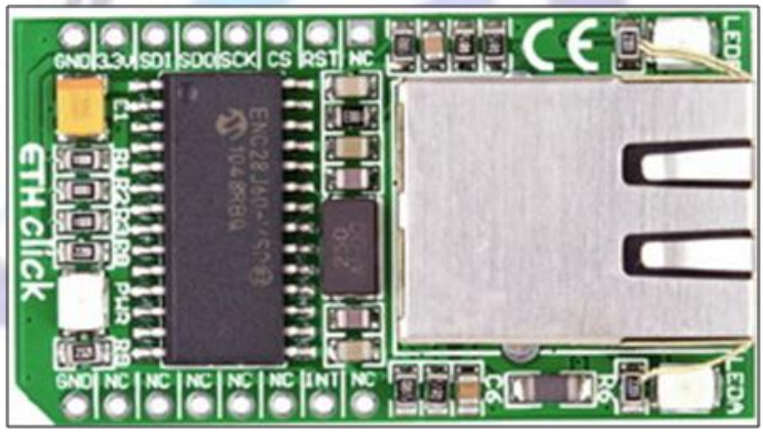

(d) ETH Click Module

Fig 4: Base station Hardware Devices

\section{End Node Hardware Platform}

\section{Microcontroller}

PIC16F88 from Microchip as shown in figure (5-a) has been used as the controller of each end node. This microcontroller has enough memory size and enough no. of I/O ports that can suite our needs in each node [5]. It processes the data received from the sensors and sends them to the serial UART interface where ZigBee can send them to Base Station. 


\section{Sensors}

Each end node has been connected with environmental sensors that are needed to be used. In our proposed system, LM35 analog temperature sensors, as shown in figure (5-b), have been used as an example and connected to one ADC channel of ADC seven channels of PIC16F88.

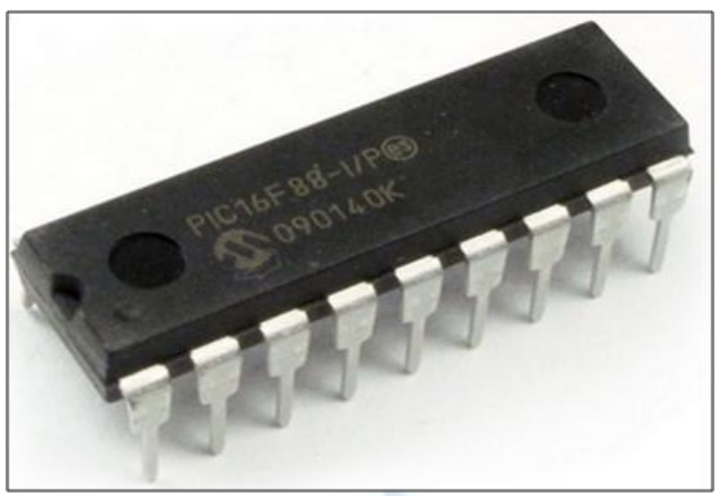

(a) PIC16F88 Microcontroller

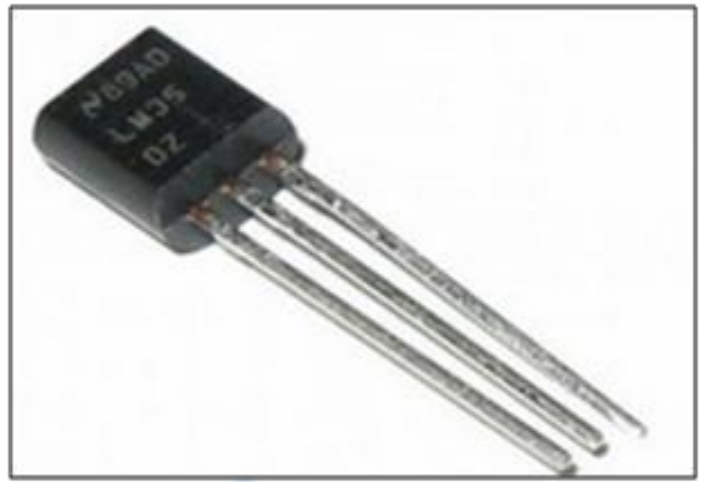

(b) Analog Temperature Sensor

Fig 5: End Node Hardware Devices

The ZigBee module has also been used in the end nodes to provide the ZigBee communication with Base Station. It has been configured as a router with the same PAN ID and same RF channel of Base Station.

\section{SYSTEM IMPLEMENTATION}

The implementation of the proposed ZigBee wireless sensor network includes: End Node and Base Station hardware and software implementation, and Host Computer Software implementation.

\section{Base Station Implementation}

Figure (6-a) shows the block diagram of Base Station. The base station has been implemented to collect end nodes sensor information and display this information on LCD and also send this information to the host computer to be saved as history information. The MCU PIC18F45K22 has been the main controller of base station operation. The MCU receives end nodes sensors information from the Coordinator ZigBee module. The ZigBee module has been connected to MCU through UART1 interface. The received information has been displayed on 2x16 LCD and also sent Ethernet controller which is connected to MCU through SPI interface.

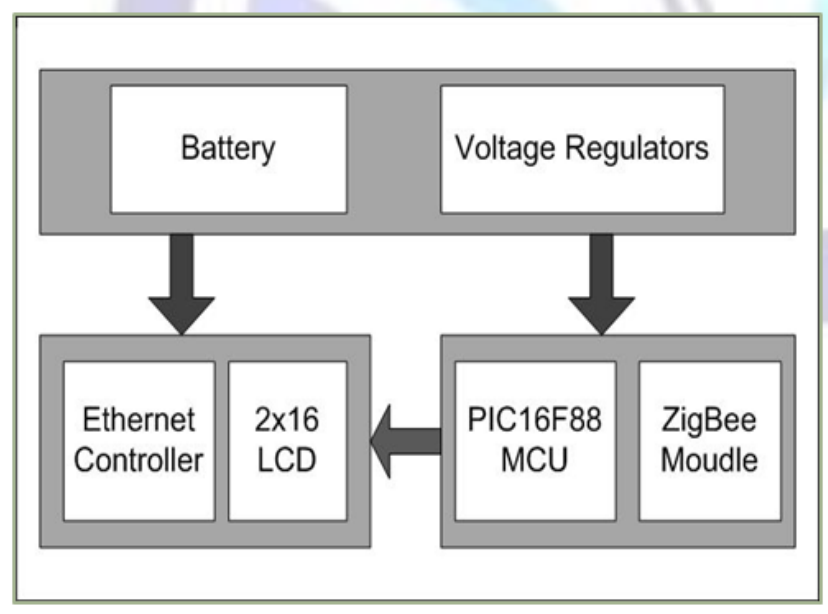

(a) Block Diagram of Base Station

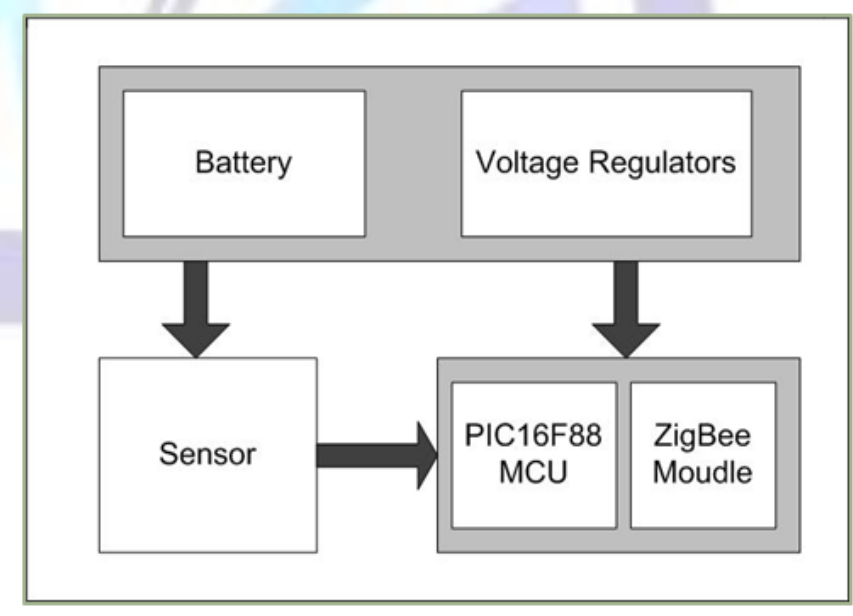

(b) Block Diagram of End Node

Fig 6: Block Diagram of Base Station and End Node 


\section{End Node Implementation}

Figure (6-b) shows the structure of proposed ZigBee end node. End node has the sensors that collect the environmental information and send this information to the microcontroller which processes the information. Analog temperature sensor has been used in the proposed system as an example. This sensor has been connected to ADC channel (1) of PIC16F88 MCU. Then the processed information has been sent to the ZigBee module. The ZigBee module sends the information to the coordinator. The ZigBee has been connected to PIC16F88 through UART interface. As a power source, each end node has been provided by DC power using $9 \mathrm{~V}$ battery and because that MCU works on DC 5V and ZigBee module works on $\mathrm{DC} 3.3 \mathrm{~V}$, voltage regulators has been used.

\section{SYSTEM OPERATION}

The proposed ZigBee Wireless Sensor Network has been composed of multiple ZigBee wireless sensor nodes, Base Station, and host computer. The flowchart for Base Station and End Nodes operation is shown in figure 7.

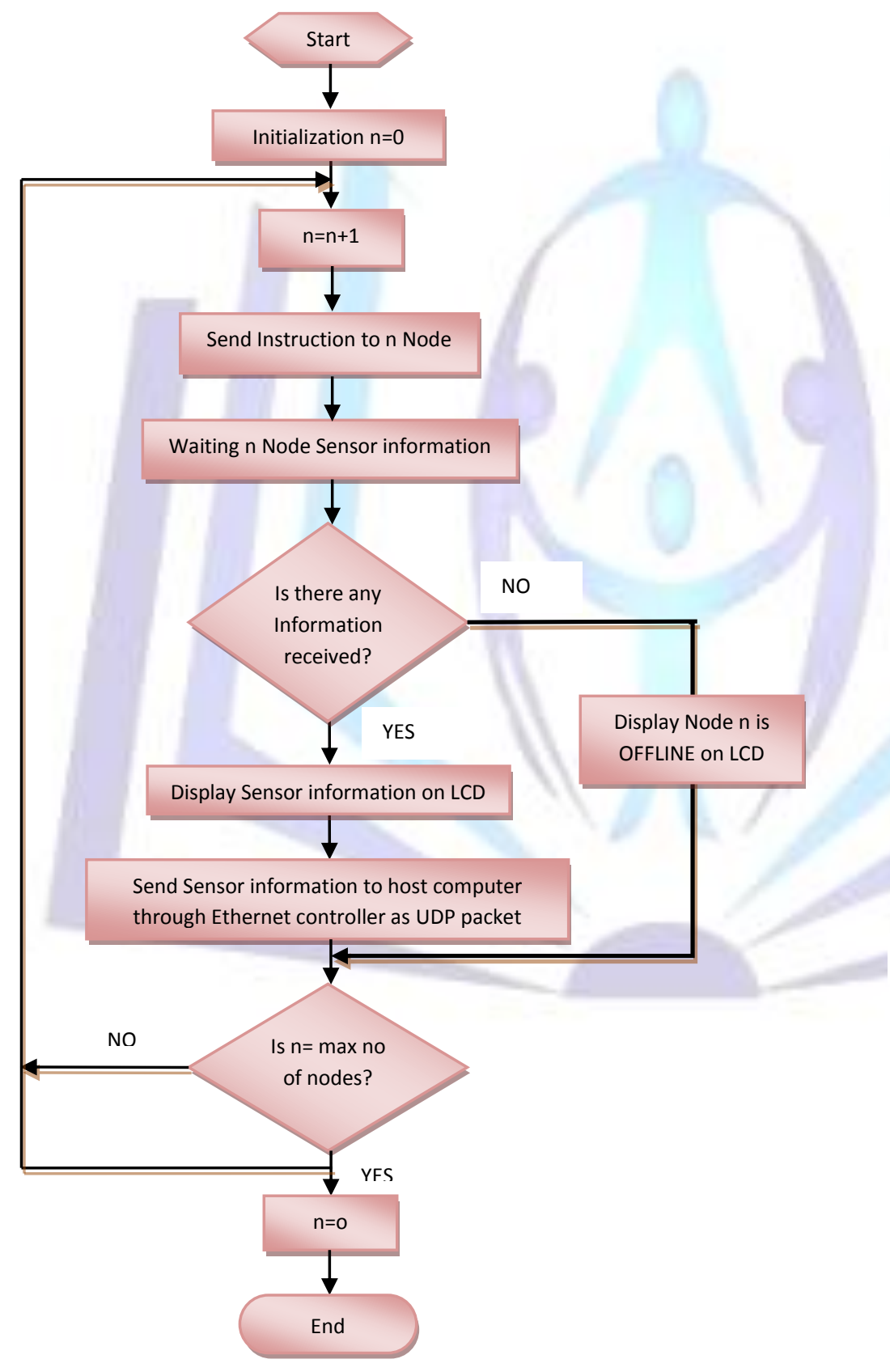

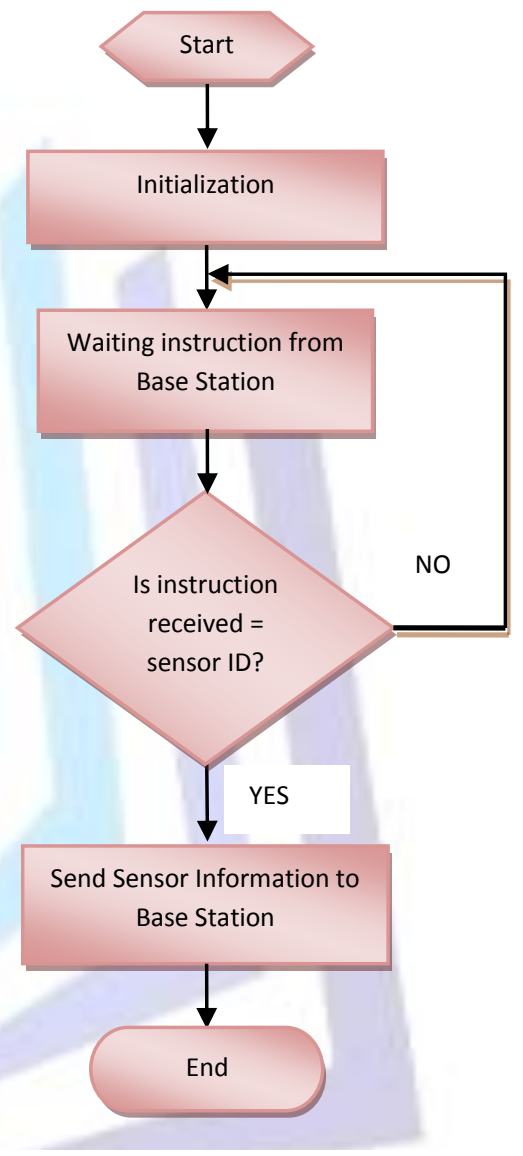

(b) End Node Flowchart

(a) Base Station Flowchart

Fig 7: Base Station and End Node System Operation 
The system operation initializes from base station. The base station sends a request to each end node and waits a specific time delay. If a node is online and receives the request, this node will get the information from temperature sensor through ADC channel and upload it to the ZigBee module. The ZigBee module sends this information to the base station coordinator. In case, the timer is finished and no information has been received from end node the base station will indicate that the end node is offline and display offline state on the LCD. Once the microcontroller of base station gets the end nodes information from the ZigBee module through UART interface, The MCU processes the information received from end nodes and sends that information to the LCD module. The LCD will display the received sensor readings. The MCU also uploads the processed sensor information of each end node to the Ethernet controller which sends this data to the host computer as a UDP packet.

The system has been deployed with a closed indoor field and nodes have been distributed on the rooms of this building. The distance between base station and single node was $10-20 \mathrm{~m}$ with successful data delivery. $20-30 \mathrm{~m}$ distance, we have got about $50 \%$ of missing readings. Exceeding $30 \mathrm{~m}$, end node lost connection with Base Station and no readings have been received. The connection has been resumed when another end node or a ZigBee router has been putted on the path between Base station and the disconnected end node as shown in figure 8. By this way, the mesh networking between ZigBee nodes has been achieved.

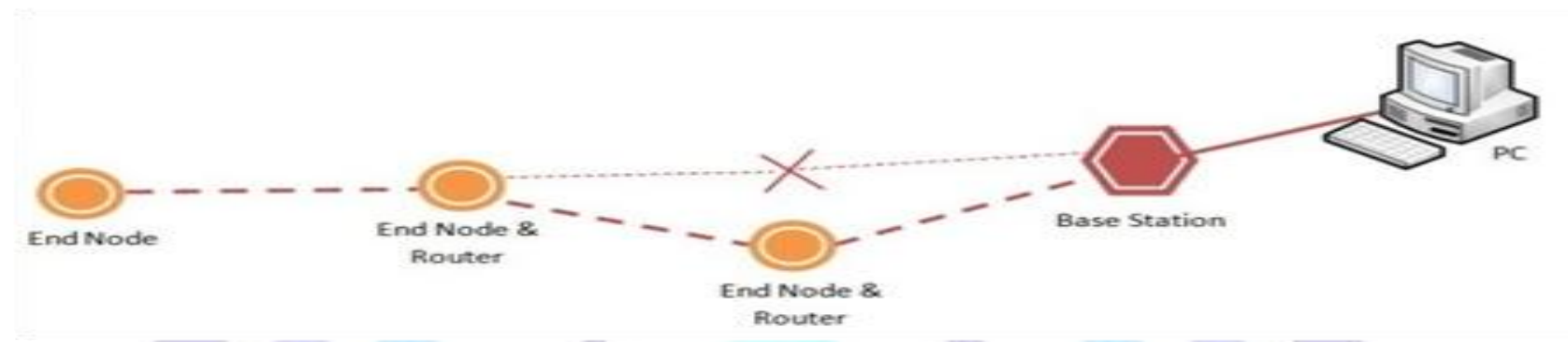

Fig 8: ZigBee Mesh Networking Test

The host computer has been used to make a data base (DB) of end nodes sensor information and this DB has been considered as a history log for sensor readings. In the host computer, a PHP code has been used to open a UDP server socket and an SQL programming code has been used to insert the received information from base station into the data base. XAMPP software application for Windows has been installed on the host computer. XAMPP has been used to install the Apache HTTP server, MySQL, PHP, and PHPMyAdmin on the host computer. Using PHPMyAdmin web-based interface tool a MySQL ZigBee DB has been built as shown in figure (8-a). The data base structure for end nodes has contained: id (record id), sensor id (Node id), value (temperature reading), and timestamp (reading received time) fields, see figure (8-b). The end nodes sensor information has been sent as UDP packets to the host computer through an Ethernet connection. This information has been inserted into the ZigBee DB using the PHP and MySQL code already running in the host computer.

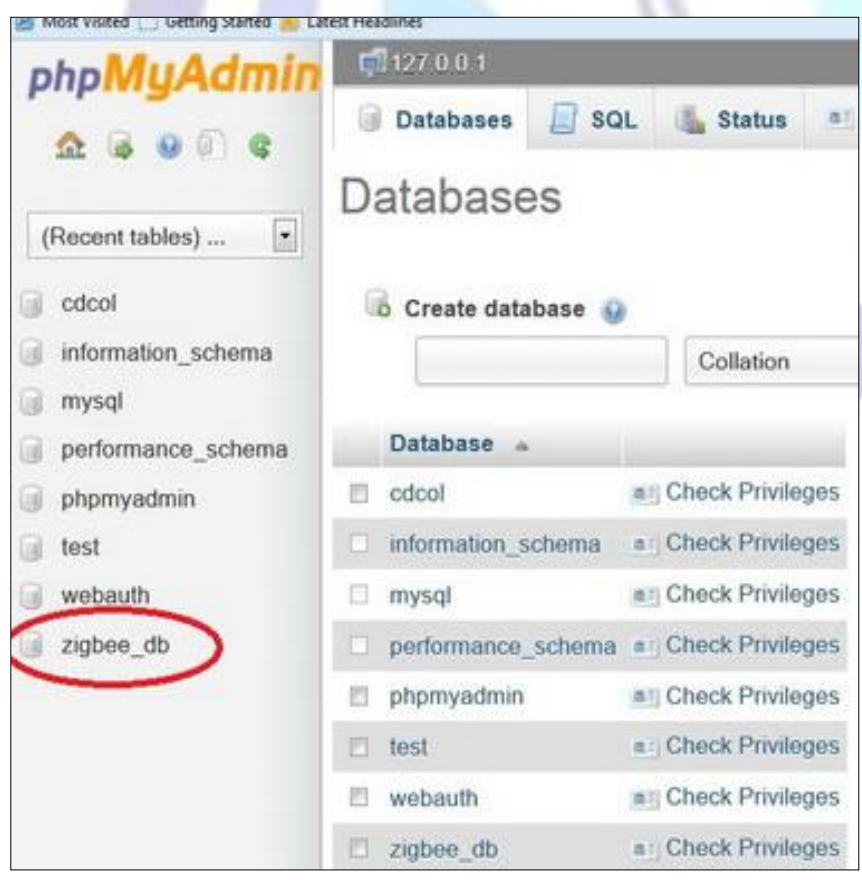

(a)Using PHPMyAdmin to create MYSQL ZigBee DB

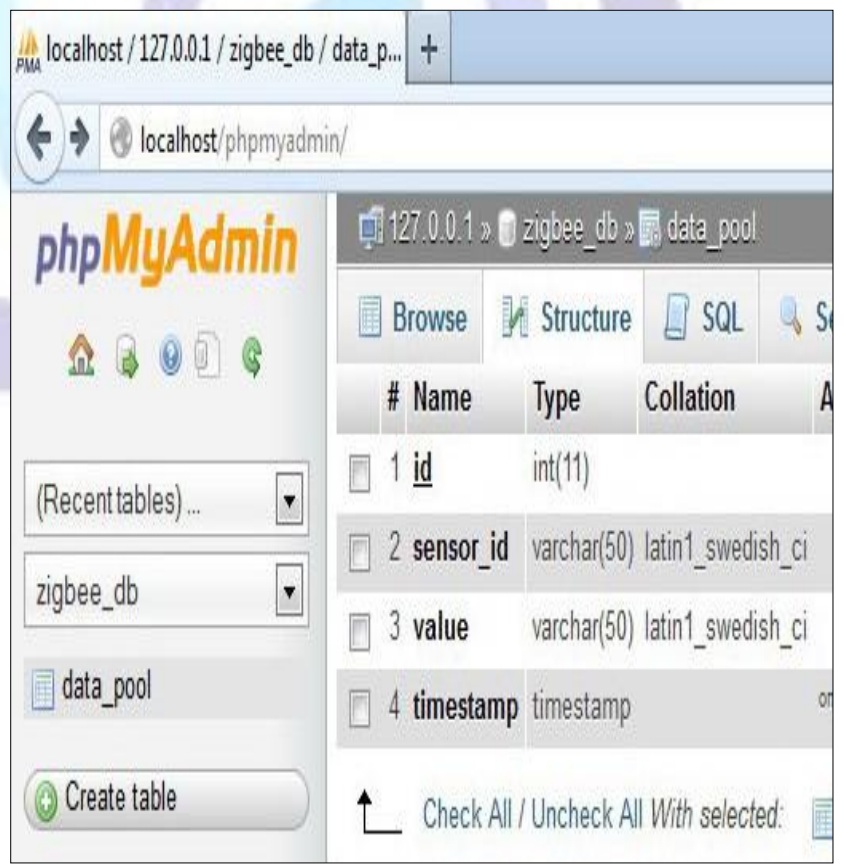

(b)DB Structure for Sensor Readings

Fig 8: Creation of ZigBee Data Base using PHPMyAdmin Web Interface 


\section{SYSTEM TESTING AND RESULTS}

The finsal hardware design for the proposed ZigBee system is shown in figure 9.

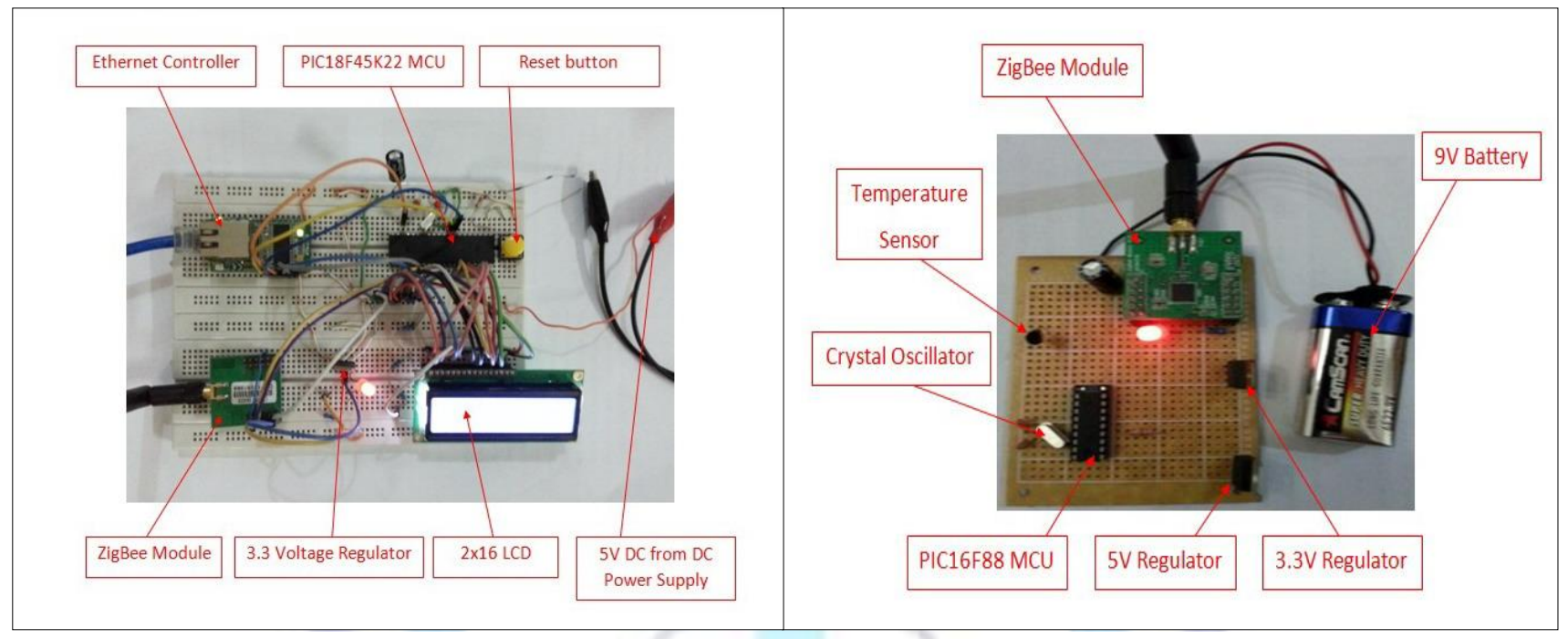

(a) Base Station

(b) End Node

Fig 9: The Final ZigBee Base Station and End Node System Hardware Design

This system has been implemented and tested with Base Station and three end nodes. The temperature sensor has been used to send the environment temperature information to the Base Station. When the system has been run in a stable fashion and end nodes located within the base station coverage, the temperature information has been received normally from the 3 end nodes and temperature information displayed on the LCD of base station as shown in figure (10-a). Whenever one of the nodes has been powered off, the offline state has been displayed on the LCD of Base Station as shown in figure (10-b).

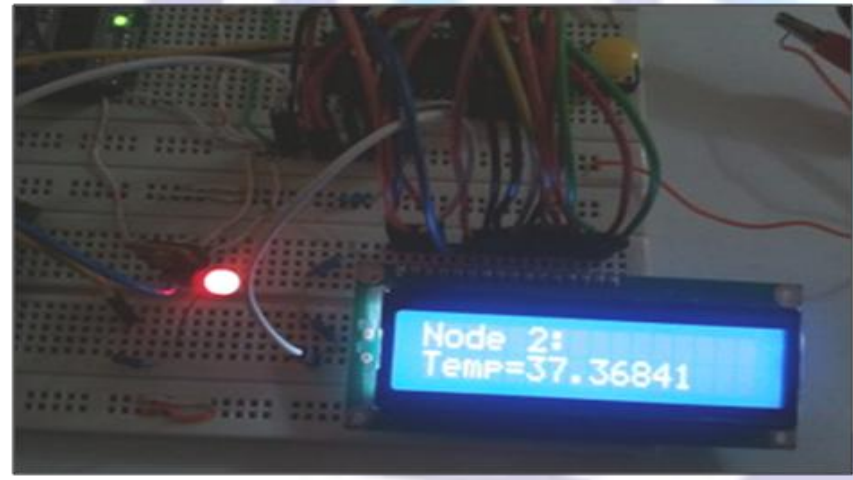

(a) Temperature reading from Node 2

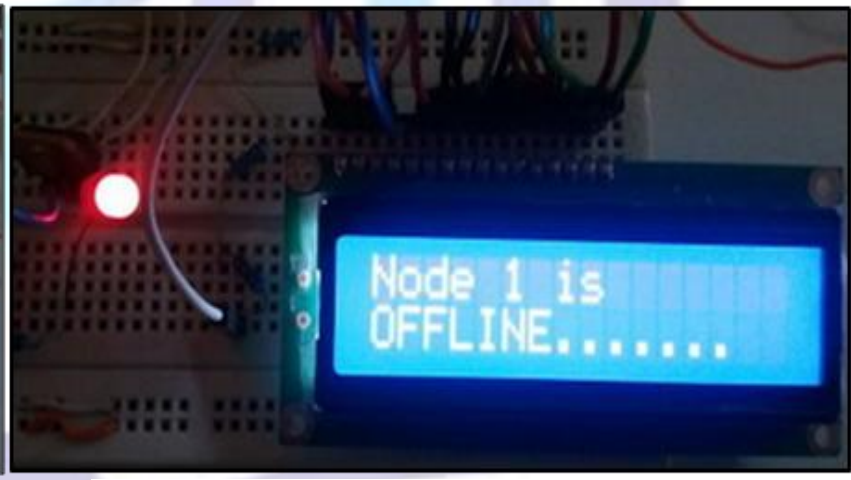

(b) Node 1 is in OFFLINE state

Fig 10: Display End Nodes information and sensor readings on Base Station LCD

Also the same information for each node has been sent to the host computer. Using XAMPP web-based server interface, the temperature readings for the 3 end nodes at different timestamps has been queried and browsed as shown in figure 11.

\section{CONCLUSION}

In this paper, a prototype of an embedded wireless sensor network has been built based on PIC microcontrollers and DRF1605 ZigBee module. The environmental temperature monitoring has been considered in this work to demonstrate the capability of applying the system. The system has the character of wireless on wiring to remove the limitation of traditional wired network system for any kind of remote conditions monitoring. 


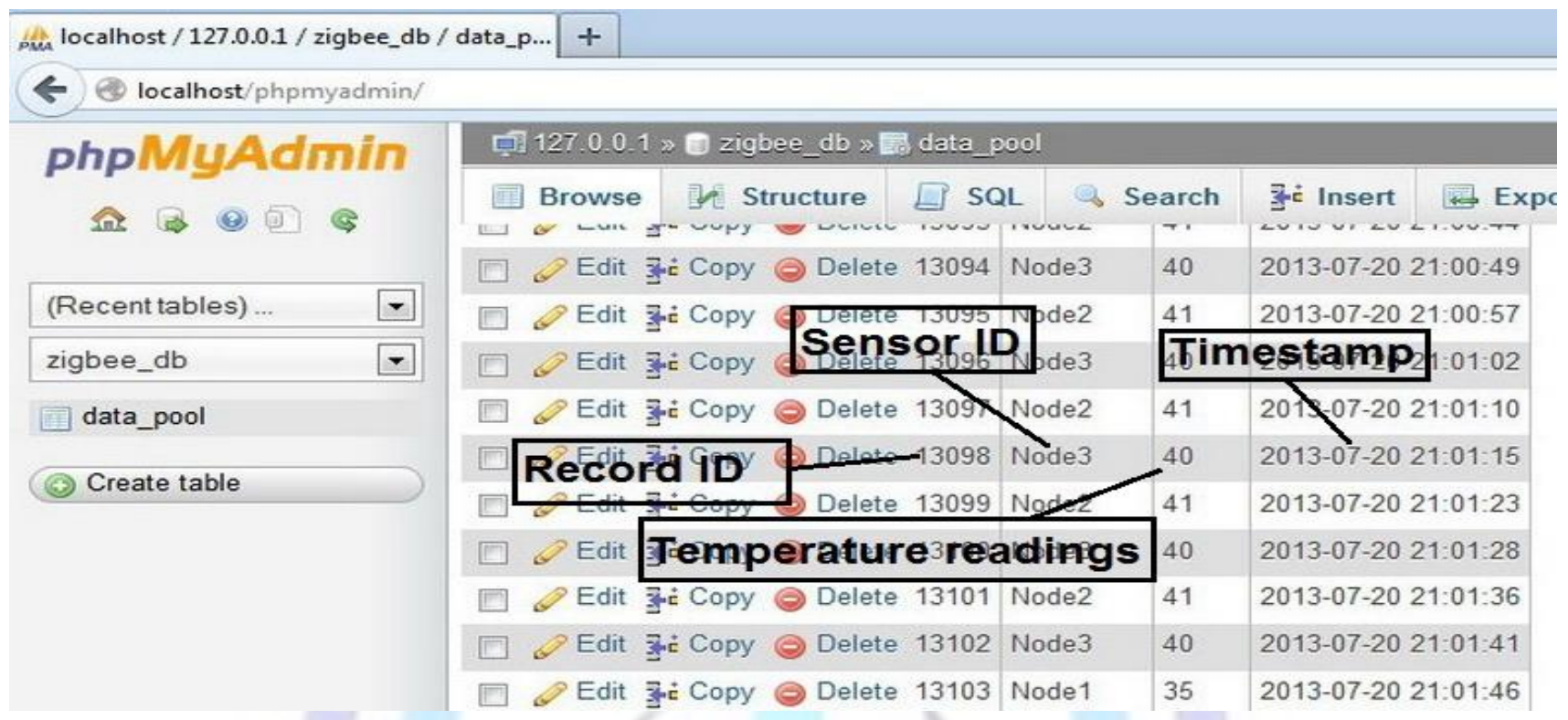

Fig 11: Temperature readings received from End Nodes and queried via web interface

\section{REFERENCES}

[1] S. Posland,Ubiquitous Computing: Smart Devices, Environments and Interactions, Wiley, 2009.

[2] W. Dargie, C. Poellabauer, and Wiley InterScience (Online service), Fundamentals of wireless sensor networks theory and practice. Chichester, West Sussex, U.K.; Hoboken, NJ: Wiley, 2010.

[3] K. Sohraby and D. Minoli, Wireless sensor networks: technology, protocols, and applications. Hoboken, N.J.: WileyInterscience, 2007Tavel, P. 2007 Modeling and Simulation Design. AK Peters Ltd.

[4] "ZigBee Specification Overview." [Online]. Available: http://www.zigbee.org/Specifications/ZigBee/Overview.aspx. [Accessed: 23-Aug-2013].Forman, G. 2003. An extensive empirical study of feature selection metrics for text classification. J. Mach. Learn. Res. 3 (Mar. 2003), 1289-1305.

[5] S. C. Ergen, "ZigBee/IEEE 802.15. 4 Summary," UC Berkeley Sept., vol. 10, 2004.

[6] J. T. Adams, "ZigBee Wireless Technology and the IEEE 802.15. 4 Radio-Enabling Simple Wireless," in Texas Wireless Symposium 2005, 2005, pp. 26-28.

[7] Microchip,"PIC18 (L) F2X/4XK22 Data sheet",Microchip.

[8] CC253x System-on-Chip Solution for 2.4-GHz IEEE 802.15.4 and ZigBee® Applications, User's Guide, Texas Instruments.

[9] DRF1605 User Guide, DTK ELECTRONICS.

[10] The DatasheetArchive - Datasheet Search Engine - Icd 2x16 HD44780 14 pin.pdf.

[11] "ETH click - Breakout board for ENC28J60 Ethernet controller." [Online]. Available: http://www.mikroe.com/click/eth/. [Accessed: 24-Aug-2013]. 